\title{
Use Cases für industrielle Anwendungen der Augmented Reality Technologie
}

\author{
Fahmi Bellalouna (D) Robin Langebach • Volker Stamer • \\ Franco Zipperling
}

Eingegangen: 12. Juli 2021 / Angenommen: 17. November 2021 / Online publiziert: 9. Dezember 2021 (C) Der/die Autor(en) 2021

Zusammenfassung Die Augmented Reality Technologie gehört zu den Schlüsseltechnologien der digitalen Transformation. Augmented Reality ist die Technologie, die die Überlagerung von physischen Objekten mit rechnergenerierten virtuellen wahrnehmbaren Daten in Echtzeit ermöglicht, um eine interaktive Anwendererfahrung in der realen Welt zu bieten. Augmented Reality wird derzeit in vielen Geschäftsbereichen wie z. B. Ingenieurwesen, Bildung, Medizin, Logistik und Transport entweder zum Experimentieren oder produktiv eingesetzt. Durch die rasante Entwicklung der Displayhardware und der mobilen Geräten im Hinblick auf die Performance als auch den Funktionsumfang ist die Realisierung von leistungsfähigen Augmented Reality Applikationen für industrielle Anwendungen einfacher geworden. Der vorliegende Beitrag wird zunächst eine kurze Übersicht über die aktuelle Entwicklung und die Bedeutung der Augmented Reality Technologie für die Industrie sowie eine Einführung in die Grundlagen der eXtended Reality und Augmented Reality Technologie geben. Anschließend werden drei Anwendungsfälle zur Entwicklung und Implementierung von Augmented Reality Anwendungen in zwei unterschiedlichen Industriebereichen - Feuerwehrtechnik und Kälte- und Klimatechnik - vorgestellt und diskutiert. Abschließend werden die im Rahmen der

\footnotetext{
Fahmi Bellalouna $(\varangle) \cdot$ Robin Langebach

Hochschule Karlsruhe, Karlsruhe, Deutschland

E-Mail: fahmi.bellalouna@h-ka.de

Robin Langebach

E-Mail: robin.langebach@h-ka.de

Volker Stamer

BITZER Kühlmaschinenbau GmbH, Rottenburg, Deutschland

E-Mail: volker.stamer@bitzer.de

Franco Zipperling

Rosenbauer Karlsruhe GmbH \& Co. KG, Karlsruhe, Deutschland

E-Mail: franco.zipperling@ rosenbauer.com
} 
Durchführung der Anwendungsfälle gewonnenen Erfahrungen mit den implementierten Augmented Reality Anwendungen zusammengefasst.

Schlüsselwörter EXtended Reality (XR) · Augmented Reality (AR) · Training • Produktkonfiguration · Montageassistent

\section{Use Cases for Industrial Applications of Augmented Reality Technology}

Abstract Augmented Reality technology is one of the key technologies of the digital transformation. Augmented Reality is the technology that enables overlaying of physical objects with computer-generated virtual perceptible data in real time to provide an interactive user experience in the real environment. Augmented Reality is currently used in many business fields such as engineering, education, medicine, logistics and transport either for experimentation or productive use. Due to the rapid development of display hardware and mobile devices in terms of performance as well as the range of functions, the implementation of powerful augmented reality applications for industrial applications has become easier. The presented paper will first give a brief overview of the current development and the importance of augmented reality technology for the industry as well as an introduction to the basics of eXtended reality and augmented reality technology. Then three use cases for the development and implementation of augmented reality applications in two different industrial areas-firefighting technology and refrigeration and air conditioning technology - will be presented. Finally, the gained project experiences during implementation and application of the use cases will be concluded.

Keywords EXtended Reality (XR) - Augmented Reality (AR) $\cdot$ Training $\cdot$ Product Configuration $\cdot$ Assembly Assistant

\section{Einführung}

Der Fortschritt bei den Informations- und Kommunikationstechnologien (IKT) eliminiert weitestgehend ökonomische Handlungseinschränkungen und schafft völlig neue Handlungsmöglichkeiten. Das führt zu Innovationen, die bislang schlicht unvorstellbar waren (Schellinger et al. 2019). Dieser Vorgang wird als , digitale Transformation“ bezeichnet und wird vom MIT Center of Digital Business als Einsatz neuer digitaler Technologien (z.B. soziale Medien, mobile Kommunikationsgeräte, integrierte Sensoren, Cloud-Computing) zur signifikanten Verbesserung und zur Steigerung der Leistung von Geschäftsprozessen (z. B. Verbesserung der Kundenerfahrung, Rationalisierung des Betriebs oder Erstellung neuer Geschäftsmodelle) definiert (Fitzgerald et al. 2014). Augmented Reality (AR) ist eine der Schlüsseltechnologien der digitalen Transformation im industriellen und nicht-industriellen Umfeld. AR ist die Technologie, die die physische Welt durch rechnergenerierte, wahrnehmbare digitale Objekte erweitert, um eine interaktive Anwendererfahrung in der realen Welt zu ermöglichen. Durch die rasante Entwicklung von Displayhardware, neuen Interaktionsgeräten und Trackingsystemen werden heute Virtual und 
Augmented Reality Anwendungen entwickelt, die vor wenigen Jahren ausschließlich in großen Forschungslaboren realisierbar gewesen wären (Dörner et al. 2013). AR hat inzwischen in vielen Branchen und Geschäftsprozessen wie z. B. Handel, Vertrieb, e-Commerce, Tourismus, Produktion, Logistik, Training, Einzug gefunden. Ein Hauptvorteil der AR-Anwendung besteht darin, Anwender in den betrachtenden Prozessen und Systemen durch hoch performante Display- und Anwendertrackingund UI-Funktionen vollständig zu integrieren und sich als Teil eines Erlebnisses zu fühlen. Der Einsatz von AR hilft dabei, räumliche Wahrnehmung von komplexen technischen und naturwissenschaftlichen Systemen im Vergleich zur einfachen 2DDesktoppräsentation zu verbessern und somit komplexe Sachverhalte greifbarer und verständlicher abzubilden. Mehrere wissenschaftliche Studien haben bewiesen, dass AR die menschlichen sensorischen Fähigkeiten fördert und die erforderliche kognitive Anstrengung reduziert, die für die Vorstellung komplexer Systeme und Produkte erforderlich sind (Liu et al. 2014; Horvat et al. 2018). Wissenschaftler der IstvánSzéchenyi-Universität in Ungarn haben die Lerneffizienzsteigerung durch AR im Hochschulbereich untersucht und sind zu folgenden Ergebnissen gekommen: $30 \%$ mehr studentische Aktivitäten und Teamarbeiten im Unterricht und $50 \%$ besseres Verständnis der Unterrichtsinhalte (Horvat et al. 2019). Das Potenzial der AR-Technologie zur Effizienzsteigerung von Geschäftsprozessen und zur Generierung von neuen Geschäftsmodellen wurde bereits von Unternehmen sowohl aus den informationstechnischen als auch aus dem industriellen Bereich erkannt. Das große Interesse von Unternehmen an AR spiegelt sich in den Marktzahlen wider. Die Marktforschungsexperten sind sich alle über das enorme Wachstumspotenzial für den ARMarkt einig. Prognosen sprechen von einem globalen AR-Marktumsatz in Höhe von 70 bis 75 Mrd. Dollar im Jahr 2023. Bis 2023 sollen bis 2,5 Mrd. AR-Geräte weltweit verkauft werden, was enorme Möglichkeiten für die Entwicklung von innovativen AR-Anwendungen schafft (DigCapital 2019). Trotz dieses Trends befinden sich die AR-Anwendungen aktuell im industriellen Umfeld häufig im Experimentierbereich und die Übertragung auf die realen Geschäftsprozesse findet in vielen Fällen noch nicht statt. Laut einer Untersuchung von Capgemini Research Institute aus dem Jahr 2018 haben sich $55 \%$ der ca. 600 befragten Unternehmen weltweit, die sich mit der AR-Technologie befassen, als Experimentator bezeichnet und $45 \%$ als Umsetzer, in Deutschland sind es $62 \%$ bzw. $38 \%$ (Capgemini 2021). Vor allem der Mangel an AR-Fachexperten in industriellen Unternehmen sowie standardisierende industrielle AR-Lösungsansätze verhindern derzeit den breiten produktiven Einsatz von AR (Nee et al. 2012).

In diesem Beitrag werden folgende drei Anwendungsfälle zur Implementierung von AR-Anwendungen im industriellen Umfeld vorgestellt:

- Feuerwehrtechnik:

- AR-Anwendung zur Konfiguration eines Rettungskorbs für das Unternehmen Rosenbauer Karlsruhe GmbH \& Co. KG.

- AR-Anwendung zur Visualisierung des Innenausbaues von Geräteräumen eines Feuerwehrfahrzeugs für das Unternehmen Rosenbauer Karlsruhe GmbH \& Co. KG. 
- Kälte- und Klimatechnik

- AR-App zur Visualisierung des Aufbaus und der Funktion eines Schraubenverdichters im Schulungs- und Trainingsbereich der Schaufler Academy des Unternehmens Bitzer Kühlmaschinenbau GmbH.

Die Anwendungsfälle wurden als Kooperationen zwischen der Hochschule Karlsruhe und den Unternehmen Rosenbauer Karlsruhe GmbH \& Co. KG und Bitzer Kühlmaschinenbau GmbH realisiert. Im Rahmen der Umsetzung der Anwendungsfälle standen folgende Fragestellungen für die beteiligten Industriepartner im Vordergrund:

- Erforderlicher Implementierungsaufwand der AR-Anwendung.

- Performance und Usability der implementierten AR-Anwendungen.

- Mehrwert und Nutzen für die eigenen Geschäftsprozesse und die Zukunftsfähigkeit der AR-Technologie.

\section{2 eXtended Reality - XR}

Die rasante technologische Entwicklung im Bereich der Computergrafik hat in den letzten Jahren zur Entstehung von einer Reihe von Anwendungen geführt, die die Realität mit der Virtualität durch Simulationstechniken kombiniert. Das Ergebnis ist die Entstehung einer künstlichen Umgebung, in der die Unterscheidung zwischen Realität und Virtualität durch gezielte Sinnestäuschung nicht möglich ist. Abhängig vom Realitäts- und Virtualitätsinhalt werden diese Anwendungen in Virtual Reality (VR), Augmented Virtuality (AV) oder Augmented Reality (AR) klassifiziert. Zur Bezeichnung der verschiedenen Anwendungsausprägungen wurde der Überbegriff eXtended-Reality (XR) eingeführt. Das „X“ wird in der Literatur ebenfalls als Platzhalter für „A“ (Augmented) oder für „,V“ (Virtual) verwendet. Der Übergang zwischen der Realität und der Virtualität wurde durch das realitäts-virtualitäts Kontinuum von Paul Milgrim abgebildet (Abb. 1) (Milgram et al. 1994). Am linken Ende des Kontinuums wird die zu betrachtende Umgebung ausschließlich real angezeigt, während eine am rechten Ende ausschließlich virtuell dargestellt wird. Angewendet auf das Beispiel mit dem Schraubenverdichter (Abb. 1) befindet sich das reale Mo-

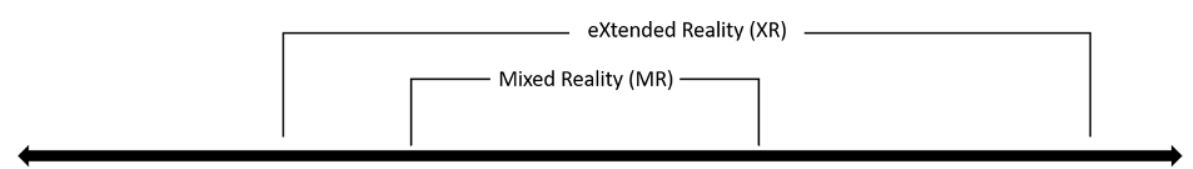

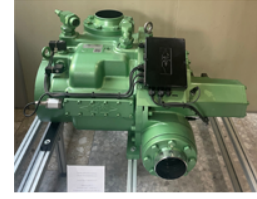

(a) Real Environment

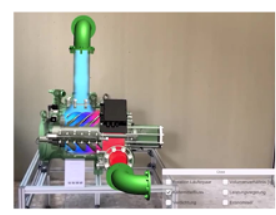

(b) Augmented Reality (AR)

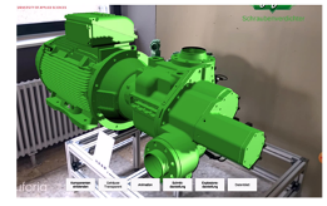

(c) Augmented Virtuality (AV)

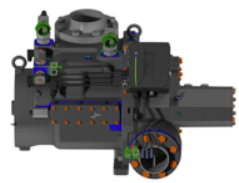

(d) Virtual Environment (VR)

Abb. 1 Realitäts-Virtualitäts Kontinuum. (In Anlehnung an Milgram et al. 1994) 
dell ganz rechts im Bereich „Real Environment“ (Abb. 1(a)) und das Grafik-Modell ganz links im Bereich „Virtual Environment“ (VR) (Abb. 1(d)) des Kontinuums. Mixed Reality (MR) ist ein Kontinuum, welches sich zwischen der Realität und der Virtualität erstreckt. Dabei nimmt der Anteil der Realität kontinuierlich ab, während sich der der Virtualität entsprechend erhöht oder umgekehrt (Dörner et al. 2013). Soweit die reale Umgebung im Fokus der Betrachtung liegt und diese durch virtuelle Inhalte erweitert wird, handelt es sich hier um eine Augmented Reality Umgebung (AR). Die virtuelle Visualisierung des Förderstrom durch den realen Schraubenverdichter stellt im beigefügten Beispiel (Abb. 1(b)) eine AR-Anwendung dar. Steht hingegen die virtuelle Umgebung im Mittelpunkt der Betrachtung und wird diese durch reale Inhalte angereicht, so lautet die Bezeichnung Augmented Virtuality (AV). Bezogen auf das illustrierte Beispiel (Abb. 1(c)) handelt es sich bei der Betrachtung und bei der Positionierung des Grafikmodells für den Schraubenverdichter und den angeflanschten Elektromotor im realen Raum auf dem Gestell um eine Augmented Virtuality Anwendung (AV).

Die Abb. 2 zeigt den Grundaufbau eines eXtended Reality Systems in Anlehnung an xr4all (xr4all 2021). Im Mittelpunkt eines XR-Systems steht die XR-Hardware, die über XR-fähige Grafik- und Rechenprozessoren verfügt. Auf der XR-Hardware werden die XR-Applikation und das Rendering ausgeführt.

Die XR-Applikation stellt die Kernanwendung zur Durchführung einer bestimmten Prozessaufgabe mit Hilfe von XR (VR, AR, AV) dar. Durch das Rendern wird die XR-Umgebung abhängig von der Betrachtungsposition und von den Anwenderinteraktionsdaten realitätsgetreu nachgebildet. Hierzu werden beispielsweise Blickwinkel, 3D-Sound und Objektbewegungen in Echtzeit gerendert. Für die Anwenderinteraktion mit der XR-Anwendung werden Input-Komponenten benötigt, die die Interaktionsdaten erfassen. Die Interaktionsdaten können visuell, auditiv, taktil oder haptisch sein. Die Datenerfassung kann mittels Sensoren wie Kameras, Mikrofone sowie Beschleunigungs- und Lagesensoren erfolgen. Die gerenderte XR-Umgebung wird mit Hilfe von Output-Komponenten ausgegeben. Dies sind Ausgabegeräte für Audio- oder visuelle Informationen wie Brillen, Bildschirme, Leinwände oder Laut-

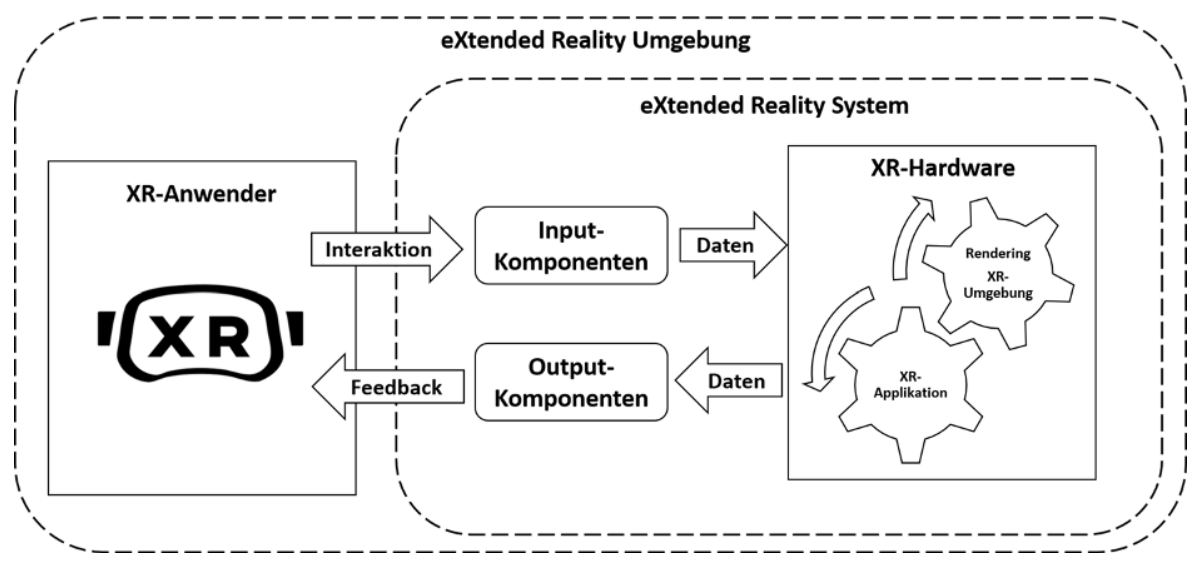

Abb. 2 Grundaufbau eines eXtended Reality Systems. (In Anlehnung an xr4all 2021) 
sprecher. Es kann aber auch eine haptische Rückkopplung mittels Force Feedback erfolgen oder die Propriozeption über eine Motion-Plattform.

\section{Augmented Reality (AR)}

Laut Dörner et al. ist AR eine - unmittelbare, interaktive und echtzeitfähige - Erweiterung der Wahrnehmung der realen Umgebung um virtuelle Inhalte - für beliebige Sinne -, welche sich in ihrer Ausprägung und Anmutung so weit wie möglich an der Realität orientiert, so dass im Extremfall eine Unterscheidung zwischen realen und virtuellen (Sinnes-) Eindrücken nicht mehr möglich ist (Dörner et al. 2013). AR erweitert die Wahrnehmung der realen Umgebung mit virtuellen Elementen, indem sie räumlich definierte digitale Inhalte in Echtzeit mit der realen Welt kombiniert. Dabei kommt es zu einer Verschmelzung der Realität mit der Virtualität (Dörner et al. 2013). Ein häufiges Beispiel für eine Anwendung von AR ist das Einfügen weiterer Informationen während der Live-Übertragung eines Fußballspiels. Abseitslinien oder Torentfernungen während eines Freistoßes werden im Fernsehen digital angezeigt (augmented-minds 2021). Theoretisch lässt sich AR aber mit jedem für den Menschen wahrnehmbaren Sinn kombinieren, z.B. visuelle AR zur Wahrnehmung von virtuellen Objekten, auditive AR zur Wahrnehmung von Geräuschen, taktile AR zur haptischen Wahrnehmung von virtuellen Objekten und vestibuläre AR zur Wahrnehmung von Bewegungen und von Beschleunigungen (Dörner et al. 2013). Zur Realisierung von AR-Anwendungen werden derzeit in der Praxis folgende gängige Methoden angewandt (Abb. 3):

- Video-See-Through AR: Bei der Video See-Through AR werden die virtuellen Inhalte über eine Videoaufnahme der realen Umgebung in Echtzeit gelegt. Mit dieser Methode ist eine Durchsicht durch das AR-Ausgabegerät nicht möglich. Als AR-Geräte können Smartphones, Tablets und Digitalkameras eingesetzt werden.

- Optical-See-Through AR: Im Rahmen der Optical-See-Through AR werden die virtuellen Inhalte auf einer durchsichtigen Projektionsfläche angezeigt. Der XRAnwender kann die reale Umgebung direkt mit den eigenen Augen wahrnehmen. Als AR-Geräte werden AR-Brillen wie z. B. HoloLens oder Magic Leap benutzt.

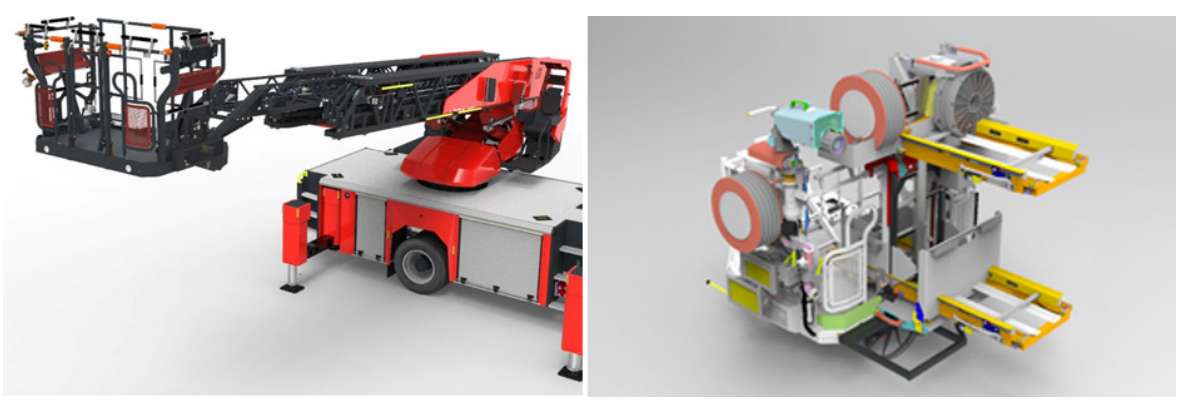

Abb. 3 Rettungskorb eines Feuerwehrfahrzeugs. (Quelle: Rosenbauer) 
- Projection-based AR: Für die Projection-based AR (Projektionsbasierte AR) werden die Oberflächen von realen Objekten als Projektionsfläche zur Abbildung virtueller Inhalte mit unterschiedlichen Farbbeleuchtungen verwendet.

\section{Einsatz der AR-Technologie im industriellen Umfeld bei den Partnerunternehmen}

\subsection{Bedeutung der AR-Technologie für die Rosenbauer Karlsruhe GmbH \& Co. KG}

XR-Technologien haben in den letzten Jahren bei Rosenbauer Karlsruhe zunehmend an Bedeutung gewonnen. Über diverse Studienarbeiten und eine Masterarbeit wurden in den letzten zwei Jahren die Möglichkeiten von VR und AR für ihren Einsatz im Unternehmen ausgelotet. Bedingt durch Corona hat insbesondere die ARTechnologie einen enormen Boost erlebt und deren Einsatz weiter vorangetrieben. Der derzeit größte Profiteur der AR-Technologie ist der Bereich Service. Durch Corona bedingte Einreiseverbote, Quarantäneauflagen nach einer Reise in Risikogebieten usw. ist die Wartung und Reparatur von Drehleitern weltweit über Nacht zu einer großen Herausforderung geworden. Als die Servicetechniker nicht mehr zu den Kunden reisen konnten, hat AR hier eine ganz neue Tür geöffnet. Durch AR konnten lokale Servicetechniker mit wenig Knowhow quasi remote durch erfahrene Servicetechniker geführt werden. Für die Zukunft wird AR auch in anderen Gebieten ihre Leistungsfähigkeit ausspielen. Dazu gehört der gesamte Bereich der Schulung sowie Marketing und Vertrieb. Im Bereich der Schulung könnte z. B. durch AR die Funktionsweise einer Feuerwehrpumpe erklärt werden. Das teure und aufwändig herzustellende Schnittmodell der Pumpe würde entfallen. Durch AR könnte das Innenleben der Pumpe, ihre Funktion und Bewegung diverser Bauteile sowie die Strömungslinien in der Pumpe visualisiert werden. Für Marketing und Vertrieb würde AR eine ganz neue Kommunikation mit dem Kunden ermöglichen. Bisher konnte dem Kunden über Fotos und Videos die Drehleiter und ihre Funktionen nahegebracht werden. Mit AR könnte sich jetzt der Kunde seine Drehleiter ,ins Wohnzimmer" stellen. Zum ersten Mal wäre es möglich, die Drehleiter zu erleben, ohne diese physisch vor Ort haben zu müssen.

\subsection{Bedeutung der AR-Technologie für die BITZER Kühlmaschinenbau GmbH}

Die Kälte- und Klimatechnik, für die BITZER wesentliche Komponenten wie Kältemittelverdichter, Wärmetauscher und Druckbehälter entwickelt, produziert und vertreibt, ist vielen Menschen aufgrund ihrer Arbeit im Hintergrund unbekannt und kann jedoch als Hidden Champion bezeichnet werden. Immerhin werden in Deutschland und auch weltweit ca. 15-18\% des Stromverbrauchs für die Kälte- und Klimatechnik benötigt Die Komponenten dürfen bzw. sollen dabei möglichst unauffällig (nicht sichtbar und hörbar) ihren Dienst verrichten, und das möglichst energieeffizient. So sorgen die Komponenten rund um den Globus für eine angenehme Frische 
und optimale Temperaturen. Um seine nicht auf Anhieb sichtbaren Produkte für die Öffentlichkeit zugänglich zu machen, begann BITZER 2009, Produkt- und Anwendungsthemen mittels Röntgenmotiven zu kommunizieren. Sie gelten als Vorläufer der aktuellen AR-Projekte. Auf der Weltleitmesse für Kältetechnik Chillventa hat BITZER im Jahr 2014 die ersten AR-Produktvorstellungen auf seinem Messestand realisiert. Und seit 2019 gibt es im neuen Headquarter des Kälte-Klima-Spezialisten in Sindelfingen einen Showroom, in dem größtenteils bewusst auf klassische, physische Schnittmodelle von Produkten verzichtet und viel mehr auf digitale Lösungen gesetzt wird. Momentan laufen in Zusammenarbeit mit der Hochschule Karlsruhe AR-Projekte, mit dem Ziel das Schulungsangebot der SCHAUFLER Academy um digitale Lehrinhalte zu erweitern. Die SCHAUFLER Academy ist das unternehmenseigene internationale Schulungs- und Trainingszentrum am BITZER Standort in Rottenburg-Ergenzingen. Darüber hinaus unterstützt BITZER den Verein Historische Kälte- und Klimatechnik e. V. mit AR-Anwendungen. Künftige AR-Projekte werden in Richtung Schulung, Service und Inbetriebnahme dieser doch recht komplexen Kälte- und Klimaanlagen gehen. Aber auch auf Messen und sonstigen Events, ob virtuell oder in Präsenz, wird AR eine zunehmende und unterstützende Rolle für die komplexen, erklärungsbedürftigen Themen des Unternehmens spielen.

\section{Anwendungsfall: AR-Anwendung zur Konfiguration eines Rettungskorbs}

\subsection{Motivation}

Ein Bild sagt mehr als tausend Worte, aber das Erleben der Realität kommuniziert mehr als jedes Foto oder Video über die Realität. Wie jetzt aber die Realität erleben lassen? Diese Frage bewegte Franco Zipperling Produktmanager bei Rosenbauer immer wieder, wenn es darum ging den Kunden die Genialität der Produkte von Rosenbauer nahezubringen. Mit dieser Fragestellung im Hintergrund ist Rosenbauer den Chancen und Möglichkeiten der AR-Technologie begegnet. Könnte es sein, dass AR genau diesen Zugang zum ,Erleben der Realität“ bieten könnte? Natürlich wäre es möglich gewesen, irgendeine AR-Anwendung zu nutzen, um einen ersten Einblick in diese Technologie zu geben. Wenn aber echte Begeisterung für AR im Unternehmen erweckt werden soll, dann ist der beste Weg dafür, die Leistungsfähigkeit von AR anhand eigener Produkte zu zeigen. Genau das war die Motivation mit der Hochschule Karlsruhe im Rahmen mehrerer Kooperationsprojekte AR-Anwendungen für die Konfiguration eines Rettungskorbs zu entwickeln. Eines der markantesten Komponenten an einer Drehleiter ist ihr Rettungskorb, welcher an der Leiterspitze montiert ist (Abb. 3). Vom Rettungskorb aus können alle Leiterbewegungen gesteuert werden. Für die Durchführung von Lösch- und Rettungseinsätzen sowie technische Hilfeleistung kann der Rettungskorb mit unterschiedlichen Zusatzausstattungen ausgerüstet werden. Hierfür stehen mehr als 50 unterschiedliche Zusatzausstattungen zur Auswahl, die an unterschiedlichen Positionen am Rettungskorb montiert sein können, die von der Feuerwehr während der Produktkonfiguration ausgewählt werden. Wie aber einem Kunden zeigen, in welcher Kombination Zusatzausstattungen für seinen 
Rettungskorb verfügbar sind und wie diese funktionieren? Dies stellt eine wesentliche Herausforderung beim heutigen Konfigurationsprozess dar. Natürlich könnten hier Fotos und Videos eingesetzt werden, um dem Kunden diese Information zu geben. Einen wesentlich direkteren Weg bietet hier die AR-Technologie. An einem vorhandenen Rettungskorb sollen über AR die unterschiedlichen Zusatzausstattungen sämtlicher Vertriebsinformationen realitätsnah visualisiert und animiert werden. Dadurch kann der Kunde direkt die ausgewählten Sonder- und Zusatzausrüstung an einem Rettungskorb interaktiv und intuitive erleben.

\subsection{AR-Anwendung zur Konfiguration eines Rettungskorbs}

Zur Unterstützung des Konfigurationsprozesses für den Rettungskorb wurde im Rahmen einer Kooperation zwischen der Hochschule Karlsruhe und Rosenbauer Karlsruhe eine AR-basierte Produktkonfigurationsanwendung entwickelt. Durch die Verwendung der AR Anwendung können die Kunden von Rosenbauer die virtuellen 3D-Modelle von den optionalen Ausstattungen innerhalb des realen Rettungskorbs mit den Grundausstattungen einblenden. Dadurch entfallen die Montage, Demontage und der Transport von den optionalen Ausrüstungen für Demonstrationszwecke im Rahmen des Vertriebs, was den Konfigurationsprozess erheblich vereinfacht und beschleunigt. Der AR-Konfigurator für den Rettungskorb wurde als Video SeeThrough AR-Applikation implementiert, die eine Verwendung mit Hilfe von gängigen mobilen Geräten (z.B. Smartphones, Tablets) als AR-Gerät ermöglicht. Im Folgenden werden die Hauptfunktionen des AR-Konfigurators beschrieben:

Modellerkennung- und -Verfolgungsfunktion verwendet eine 2D-Projektionsansicht als Target-Modell zur Erkennung des realen Rettungskorbs durch die Kamera des AR-Geräts (Abb. 4). Sobald das reale Modell erkannt wird, wird das virtuelle 3D-Modell eingeblendet und dem realen Rettungskorb überlagert. Diese Funktion ermöglicht ein unterbrechungsfreies Modeltracking auch während der Anwenderbe-

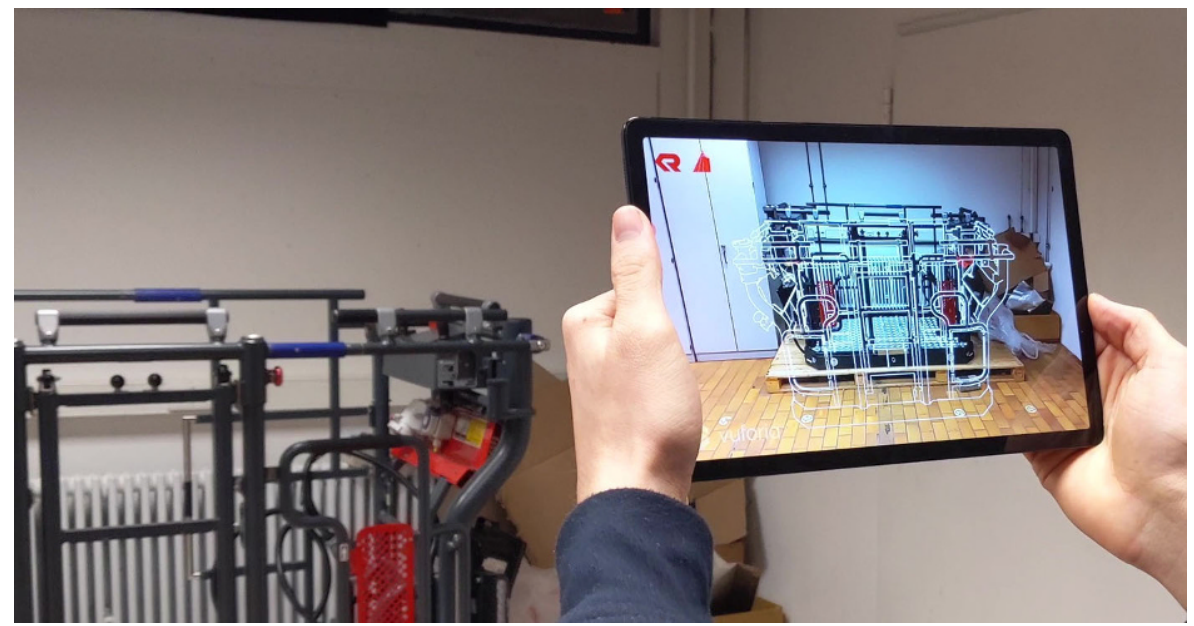

Abb. 4 Modellerkennung mit dem AR-Target-Modell 


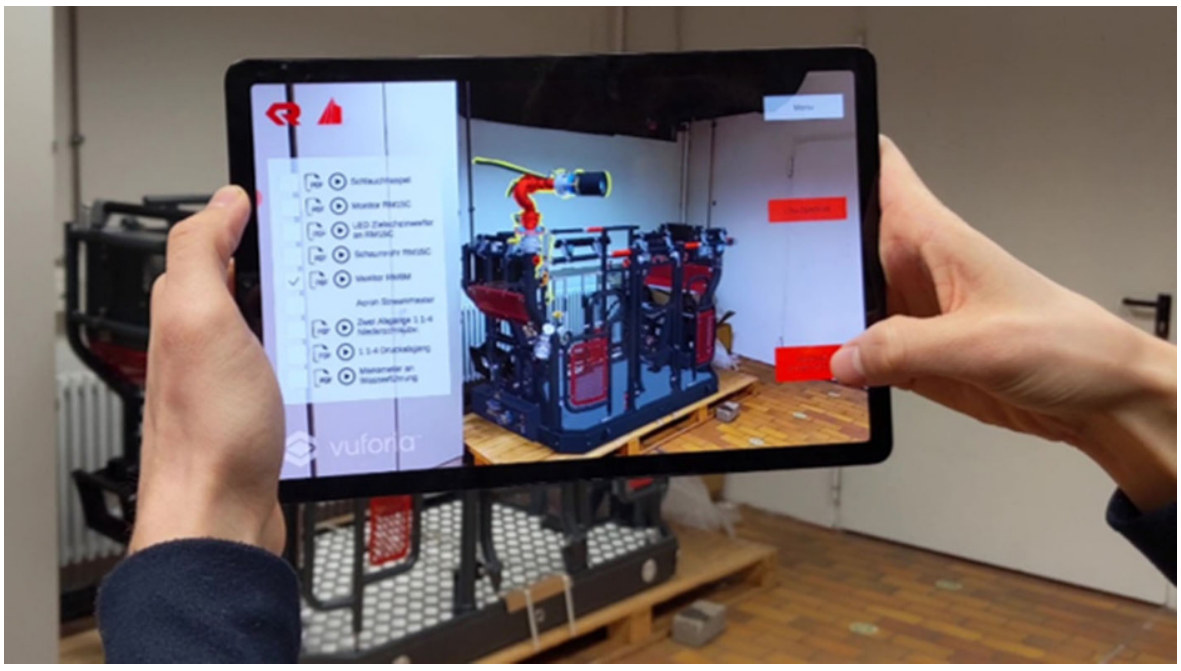

Abb. 5 AR-Funktionen zur Strukturdarstellung und zum Ein-/Ausblenden von virtuellen Daten

wegung im Raum. Die Erkennung des Grundmodells des Rettungskorbs, basierend auf Katalogillustrationen, kann auch durch diese Funktion erreicht werden.

Funktion zur Strukturdarstellung der optionalen Ausstattungen des Rettungskorbs in Form einer Baumstruktur geteilt in mehreren Kategorien (z. B. Sicherheit, Löschsystem, Kamerasystem) (Abb. 5). Damit kann der Kunde im virtuellen Ausstattungskatalog leicht navigieren und nach den benötigten Komponenten systematisch und intuitiv suchen.

Funktion zum Einblenden der virtuellen Daten ermöglicht die Anzeige der virtuellen 3D-Modelle einer oder mehrerer ausgewählter Komponenten in der Baumansicht in der richtigen Position innerhalb des realen Rettungskorbs (Abb. 5). Diese Funktion enthält Konfigurationsregeln, die die Auswahl widersprüchlicher Komponenten verhindert. Die Trackingfunktion erlaubt die Betrachtung des angezeigten 3D-Modells aus mehreren Perspektiven zur Kollisions- und Montierbarkeitsprüfung.

Funktion zur Bewegungsanimation dient dazu, die ausgewählten Ausstattungen aus der Baumstruktur innerhalb des realen Rettungskorbs dreidimensional und realitätsgetreu zu animieren. Mit dieser Funktion kann sich der Kunde einen realistischen Eindruck von den verbauten Ausrüstungen verschaffen und ferner eine Kollisionsprüfung mit den benachbarten Komponenten erzeugen. Somit wird ein wichtiger Beitrag zur Gewährleistung eines zuverlässigen und effizienten Konfigurationsprozesses gewährleistet.

Funktion zum Laden technischer Dokumente wird benutzt um technische Unterlagen der verbauten Ausstattungen während der Rettungskorbkonfiguration als PDF zu öffnen. Damit braucht der Kunde das Produkthandbuch nicht durchzublättern, um nach der technischen Spezifikation der ausgewählten Komponenten zu suchen. Dies spart wertvolle Zeit beim Konfigurationsprozess und Fehler werden bei der Verwendung von falschen Dokumenten vermieden. 
Funktion zum Abspielen von Videos ermöglicht dem Kunden während des Konfigurationsprozesses Demonstrationsvideos zum Einsatz der ausgewählten Ausstattungen in der realen Umgebung zu laden und abzuspielen. Diese Funktion bietet eine wertvolle Unterstützung für den Kunden, um die richtige Auswahlentscheidung zu treffen.

\subsection{Evaluierung der AR-Anwendung zur Konfiguration eines Rettungskorbs durch Rosenbauer}

Für die Erstellung der AR-App zur Konfiguration eines Rettungskorbs wurden einige Sonderausstattungen ausgewählt, die in AR in einen vorhandenen Rettungskorb visualisiert und konfiguriert werden sollten. Während der Entwicklung der AR-App hat sich gezeigt, welchen Stellenwert der Bedienerführung über diverse Menüs und somit der Benutzererfahrung (UX) zukommt. Die Implementierung von Konfigurationsregeln z.B. zum gegenseitigen Ausschließen von Sonder- und Zusatzausstattungen stellt eine große Untersetzung zur Vermeidung von Konfigurationsfehlern dar. Als weitere, wertvolle Funktion hat sich die Möglichkeit herausgestellt, in AR auch Bewegungen simulieren zu können. Z. B. die Bewegung eines Wasserwerfers auf dem Rettungskorb oder das Herunterklappen der Rampe, um mit einem Rollstuhl in den Rettungskorb zu fahren. Hierdurch wurde die Funktionsweise dieser Zusatzausstattung eindrucksvoll kommuniziert.

\section{Anwendungsfall: AR-Anwendung zur Visualisierung des Innenausbaues von Geräteräumen eines Feuerwehrfahrzeugs}

\subsection{Motivation}

Die Feuerwehrausrüstungen (z.B. Motorsäge, Axt, Schläuche, Feuerlöscher, Verbandkasten) sind in verschiedenen Geräteräumen in einem Feuerwehrauto untergebracht (Abb. 6). Es gibt eine Vielzahl von Ausrüstungskonfigurationen, die in den

Abb. 6 Geräteräume für Feuerwehrausrüstungen. (Quelle: Rosenbauer)

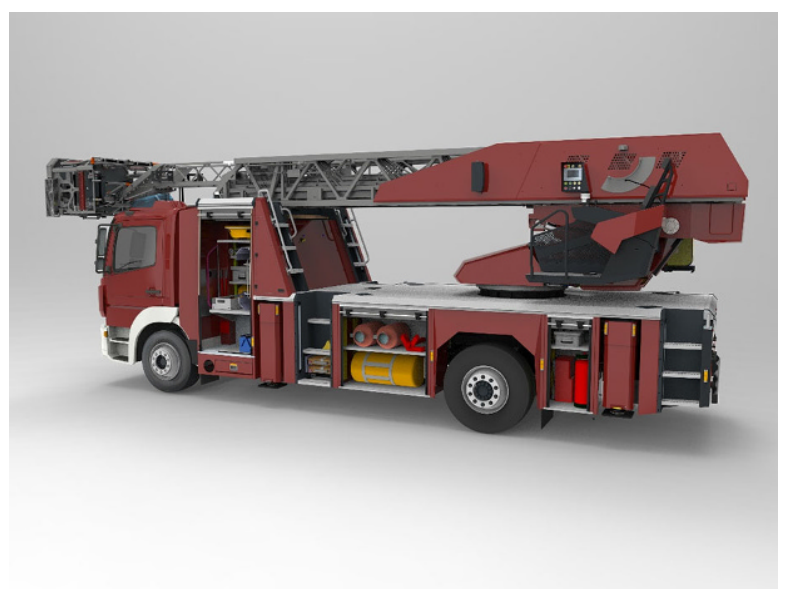


verschiedenen Geräteräumen eines Feuerwehrfahrzeugs mit unterschiedlichen Positionen verstaut sind. Diese Konfigurationen werden während des Vertriebsprozesses durch die Feuerwehren individuell festgelegt, mit dem Ziel die Ausrüstungsgegenstände optimal in den verschiedenen Geräteräumen zu platzieren. Die Festlegung und die Planung der Ausrüstungen wird bereits seit geraumer Zeit in 3D durchgeführt. Dies soll für die Montagemitarbeiter in Form einer AR-Anwendung bereitgestellt werden. Mit Hilfe der AR-Technologie sollen Geräteräume sämtliche Ausstattungen virtuell dargestellt werden. Damit soll dem Mitarbeiter, der den Innenausbau vornimmt, gezeigt werden, wo welche Komponenten verbaut werden sollen. Dadurch wird der Mitarbeiter in seiner Tätigkeit unterstützt und Fehler werden beim Umsetzen des Innenausbaus wesentlich reduziert.

\subsection{AR-Anwendung zur Visualisierung des Innenausbaus von Geräteräumen eines Feuerwehrfahrzeugs}

Um die Qualität zu verbessern und die Fehlerquote während des Montageprozesses zu reduzieren, wurde ein AR-basierter Montageassistent in Zusammenarbeit mit der Hochschule Karlsruhe und dem Unternehmen Rosenbauer Karlsruhe GmbH \& Co. KG entwickelt. Der Montageassistent wurde als Video See-Through AR-Anwendung entwickelt und dient hauptsächlich dazu, die Feuerwehrausrüstungen virtuell in realen Geräteräumen eines Feuerwehrfahrzeugs zu visualisieren. Zur Benutzung der AR-Anwendungen können marktübliche Mobilgräte (z. B. Tablet, Smartphone) eingesetzt werden. Dies soll neuen Mitarbeitern den Einstieg in den Montageprozess erleichtern und sie während der Ausrüstung von Feuerwehrfahrzeugen unterstützen. Darüber hinaus kann der AR-Montageassistent verwendet werden, um den Endmontagezustand am realen Feuerwehrfahrzeug in der frühen Verkaufsprozessphase virtuell zu visualisieren. Dies führt zu Reduzierung von Missverständnissen zwischen Kunden und Hersteller während der Auftragserstellung. Die AR-Anwendung zur Visualisierung des Innenausbaues von Geräteräumen eines Feuerwehrfahrzeugs funktioniert wie folgt:

Zum Start des AR-Montageassistenten wird der QR-Code, der einen auftragsspezifischen Feuerwehrausrüstungsgeräteraum darstellt, gescannt. Basierend auf die QR-Codedaten, werden die virtuellen 3D-Daten des Geräteraumes geladen. Danach wird durch die Kamera des AR-Gerätes mit Hilfe eines Target-Modells der betroffene Geräteraum im realen Feuerwehrauto identifiziert und getrackt. Nach der Identifizierung des realen Raums werden die virtuellen 3D-Modelle des Raumes und die dazugehörigen Feuerwehrausrüstungen an den richtigen Positionen angezeigt (Abb. 7). Die Kamera des AR-Geräts gewährleistet hierzu ein ununterbrochenes Modell-Tracking, auch wenn sich der Benutzer im Raum um den Gerätekasten bewegt. Außerdem werden die Feuerwehrausrüstungen als Baumstruktur in der ARAnwendung dargestellt, die das Ein- und Ausblenden im 3D-Raum ermöglicht. Zusätzlich zu den 3D-Modellen können weitere Daten wie z. B. Gerätenummer und Gerätename aufgerufen werden. Mit den implementierten AR-Funktionen können die Mitarbeiter die virtuellen 3D-Modelle der Ausrüstungen interaktiv während des Montageprozesses visualisieren, um einen besseren Überblick über den Geräteraum zu erhalten, bevor sie mit der Montage beginnen. Darüber hinaus kann die AR-An- 


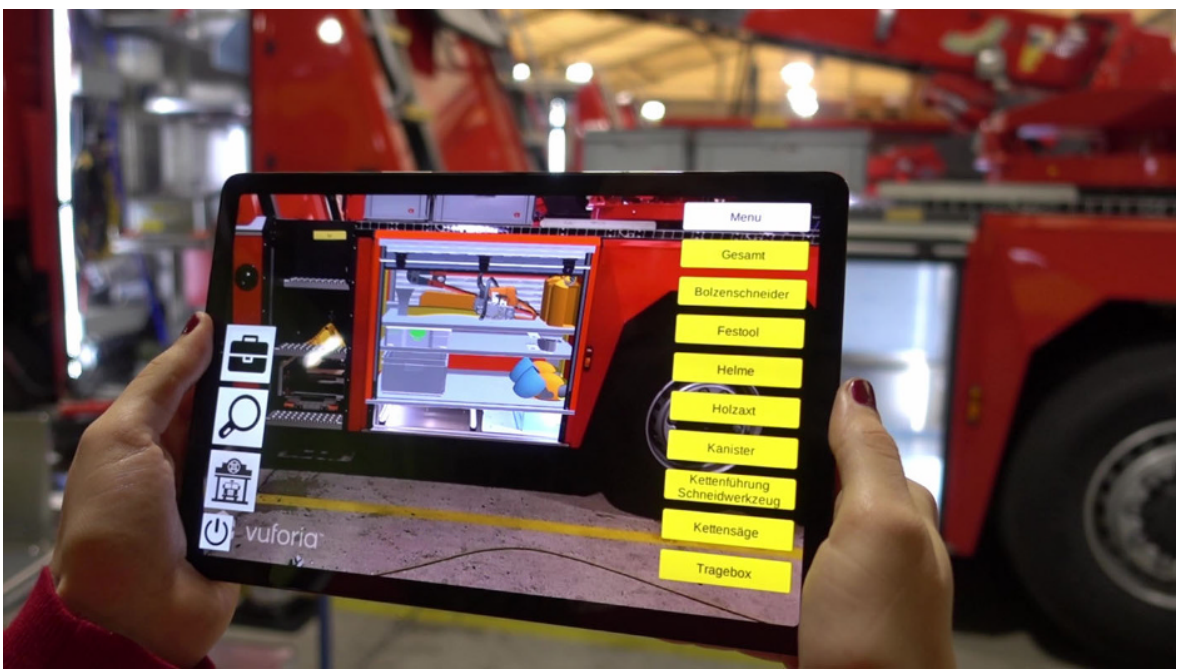

Abb. 7 AR-Anwendung zur Visualisierung des Innenausbaus von Geräteräumen eines Feuerwehrfahrzeugs

wendung auch verwendet werden, um die Qualität des Montagevorgangs am Ende hinsichtlich der Richtigkeit und der Vollständigkeit der Ausstattungen zu prüfen.

\subsection{Evaluierung der AR-Anwendung zur Visualisierung des Innenausbaus von Geräteräumen eines Feuerwehrfahrzeugs durch Rosenbauer}

Für die Erstellung der AR-App zur Visualisierung des Innenausbaus von Geräteräumen eines Feuerwehrfahrzeugs wurden einige Ausrüstungsgegenstände ausgewählt, die in AR in einen vorhandenen Gerätekasten eingeblendet werden sollten. Wie schon bei der AR-App für den Rettungskorb hat sich auch hier die Wichtigkeit der Menüführung und der Usability herausgestellt. Auch diese AR-Anwendung ist auf sehr gute Resonanz bei den Anwendern gestoßen. Am Ende hat sich jedoch noch ein anderer Nutzerkreis erschlossen. Die Gerätekästen mit Beladung können auch für sich allein aufgerufen werden. Dadurch ergibt sich die Möglichkeit, dem Kunden den Innenausbau seiner Gerätekästen per AR-App zur Verfügung zu stellen.

\section{Anwendungsfall: AR-App zur Visualisierung des Aufbaus und der Funktion eines Schraubenverdichters im Schulungs- und Trainingsbereich}

\subsection{Motivation}

Die in Kälte- und Klimaanlagen eingesetzten Komponenten haben ein Innenleben, das sich nicht unbedingt von selbst erschließt. Aber genau im Inneren und durch Optimierung der Funktionsweise und Kreisprozesse unterscheidet sich massiv, ob kli- 
Abb. 8 Offener Schraubenverdichter der OS.A95-Serie des Unternehmens BITZER

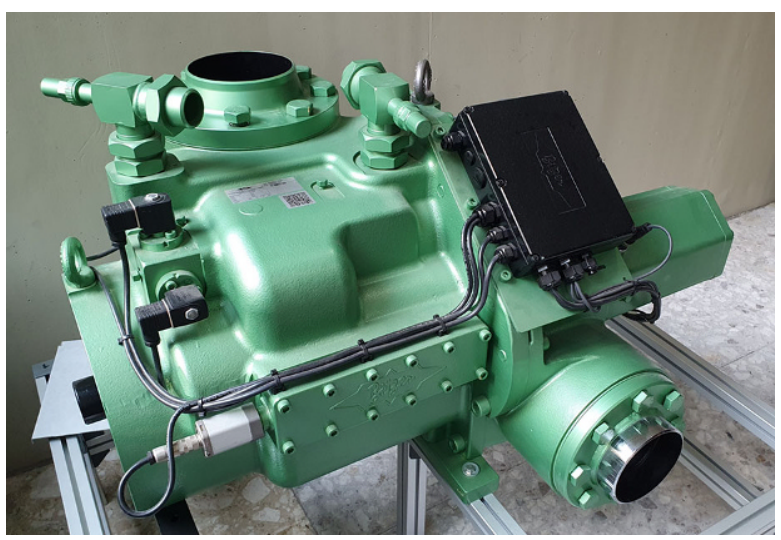

mapolitische Ziele wie Erhöhung der Energieeffizienz und Verringerung des Carbon Footprint, erreicht werden können oder nicht. Um diese recht komplexen Maschinen zu erklären, gibt es aufwändig gebaute Schnittmodelle, die ein Blick ins Innenleben ermöglichen. Solche Schnittmodelle befinden sich zu Schulungszwecken auch in den Räumlichkeiten der SCHAUFLER Academy. Sie erfreuen sich großer Beliebtheit und stoßen bei den Besuchern stets auf viel Interesse - für die Produkte von BITZER im Speziellen und die Kälte-Klima-Branche im Allgemeinen. Der große Vorteil von realen Schnittmodellen ist, dass sie Technik anfassbar und physisch erlebbar machen. Als Nachteile können die Kosten für die Erstellung solcher Modelle gesehen werden, zudem der hohe zeitliche Aufwand, der aufgebracht werden muss, die einmal erstellten Modelle kontinuierlich an den aktuellen Entwicklungsstand anzupassen. Darüber hinaus geht der Trend hin zu größeren Leistungen und damit größeren Maschinen, beispielsweise für District Cooling und Heating. Die Folge: Die Modelle werden in der Handhabung zunehmend unhandlich. Was noch dafür spricht, dass BITZER künftig verstärkt auf AR-Anwendungen setzt: In den letzten Jahren hat die Ausbildungswerkstatt am Unternehmensstandort in RottenburgErgenzingen die Schnittmodelle gefertigt. Aufgrund von Lehrplanänderungen ist dies seit geraumer Zeit nicht mehr möglich. Des Weiteren verfügen die auf Messen eingesetzten BITZER Verdichter aus Gewichtsgründen nicht über das tatsächliche Innenleben. Sie verkörpern lediglich die äußere Hülle. Ein solches, mittlerweile ausgedientes Messemodell eines sogenannten offenen Schraubenverdichters (Abb. 8) wurde der Hochschule Karlsruhe mit dem Ziel übergeben - ein erstes AR-Projekt in Zusammenarbeit mit BITZER durchzuführen, welches das komplette Innenleben des Verdichters mittels AR darstellen soll.

\subsection{AR-Anwendung zur Visualisierung des Aufbaus und der Funktion eines Schraubenverdichters im Schulungs- und Trainingsbereich}

Im Rahmen der Kooperation zwischen der Hochschule Karlsruhe und der Schaufler Academy des Unternehmens BITZER Kühlmaschinenbau GmbH wurde eine AR Anwendung entwickelt, die den Nutzen und das Potenzial der AR-Technolo- 
gie für das Lehren und die Vermittlung von komplexen technischen Systemen im Bereich der Kälte- und Klimatechnik im Schulungs- und Trainingsbereich aufzeigen soll. Der Schraubenverdichter bietet hierfür ein geeignetes Anwendungsbeispiel, da er komplexe Funktionen besitzt, welche oftmals nicht durch 2D-Unterlagen, wie zum Beispiel Bücher, Schulungsunterlagen oder Videos, ersichtlich gemacht werden können. Schraubenverdichter werden in der Kältetechnik typischerweise für große Fördervolumenströme bei geringen bis mittleren Druckverhältnissen eingesetzt. Dabei bilden die schraubenförmig gestalteten Läufer beim Eingriff einer Zahnflanke des ersten Läufers in den Zahnzwischenraum des zweiten Läufers einen Arbeitsraum aus, welcher durch kontinuierliche Rotation zur Druckseite hin verkleinert wird. Bauartbedingt besitzen diese Schraubenverdichter daher ein eingebautes Volumenverhältnis, welches durch die druckseitige Steuerkante beeinflusst wird. Darüber hinaus ergibt sich bauartbedingt - je nach geometrischer Gestaltung der Läuferquerschnitte - ein sogenanntes Blasloch. Diese unerwünschte geometrische Öffnung erlaubt einen Rückstrom von verdichtetem Kältemittel zurück zur Saugseite. Um die damit einhergehenden Effizienzverluste durch innere Leckage zu minimieren, werden Schraubenverdichter mit einem hohen Ölanteil im Fördervolumenstrom betrieben. Das Verständnis für die Ausbildung des Arbeitsraums sowie die Lage des Blaslochs sind selbst mit einem klassischen Schnittmodell nur äußerst mühsam darstellbar und zu vermitteln. Eine AR-Anwendung, die die computergenerierte Baugruppenstruktur und Funktionsweise des Schraubenverdichters über eine 2D-Abbildung (z. B. Produktkatalog, Produktflyer) oder ein physisches geschlossenes Model (z.B. das reale Schraubenverdichter-3D-Druckmodell) überlagert und visualisiert, soll den Aufwand durch die Fertigung von Schnittmodellen für Schulungs- und Trainingszwecke erheblich reduzieren. Ferner soll die Anwendung ein AR-Erlebnis anbieten, das die Lerneffizienz durch eine stärkere Einbindung der Trainingsteilnehmer verbessern soll und damit das gelernte Wissen nachhaltig abgespeichert werden kann. Die entwickelte AR-Anwendung wurde als Video See-Through Applikation realisiert und kann über ein mobiles iOS- und Android-Gerät verwendet werden. Um die Funktionalitäten des Schraubenverdichters interaktiv und intuitiv erkunden zu können, werden in der AR-Anwendung sechs Hauptfunktionen realisiert:

Funktion für Komponenten Ein-/Ausblenden der virtuellen Daten ausgewählter Komponenten des Schraubenverdichters. Zudem kann das Gehäuse transparent geschaltet werden, wodurch die innen liegenden Komponenten des Schraubenverdichters sichtbar werden.

Funktion für Schnittdarstellungen zur Visualisierung von verschiedenen Schnittansichten, um die Innenbaugruppenstruktur des Schraubenverdichters virtuell auf Basis des realen Modells darzustellen (Abb. 9). Die Schnitte können entweder statisch als Teil-/Halbschnitt oder dynamisch und somit individuell in allen Koordinatenachsen ausgewählt werden.

Animationsfunktion zur Darstellung der komplexen Hauptfunktionen des Schraubenverdichters wie z.B. Kältemittelfluss, Rotation des Läuferpaares, Verdichtungsvorgang, Leistungsregelung in greifbarer und verständlicher Art und Weise (Abb. 10). Die Animationen können teilweise interaktiv gesteuert werden. 


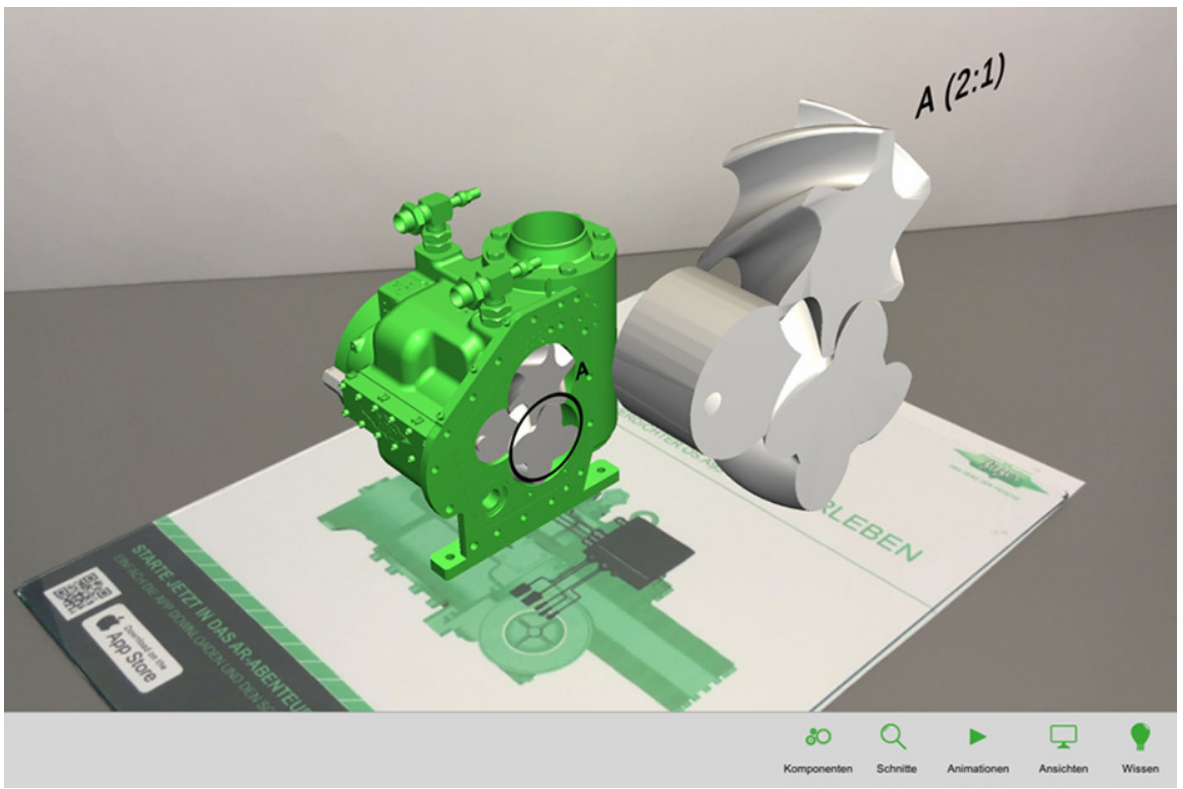

Abb. 9 AR-Funktion für die Schnittdarstellung

Abb. 10 AR-Animationsfunktion des Kältemittelflusses

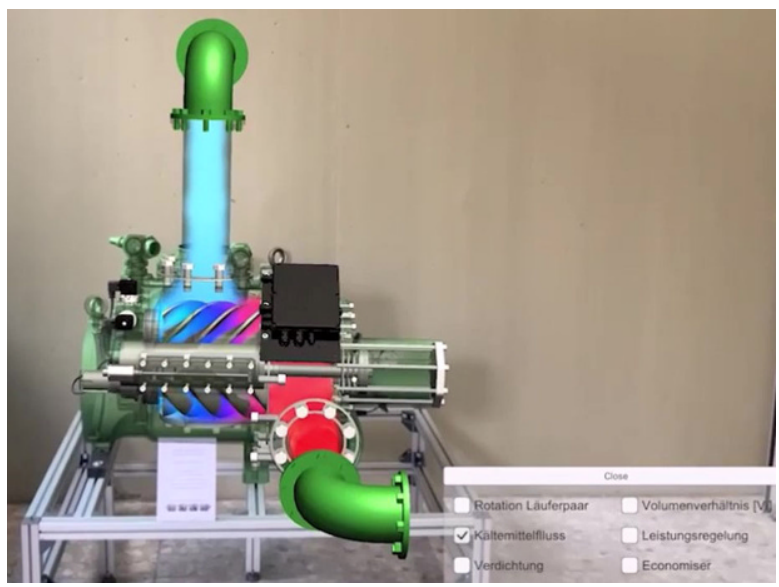

Über die Funktion Ansichten können spezielle Ansichten wie z.B. die Explosionsdarstellung (Abb. 11) oder die Ansicht des Verdichtungsarbeitsraums ausgewählt und teilweise interaktiv beeinflusst werden.

Funktion zur kontextabhängigen Informationsdarstellung erweitert die angezeigten virtuellen 3D-Modelle der ausgewählten Komponenten durch weitere Informationen aus der Fachliteratur wie z.B. Textbeschreibungen, Einsatzdiagramme, die teilweise animiert sind (Abb. 12). Diese Informationen werden abhängig von der in der Anwendung gewählten Ansicht kontextabhängig angezeigt. Dies soll dazu dienen, die angezeigten Inhalte im Rahmen der AR-Anwendung besser zu verstehen. 


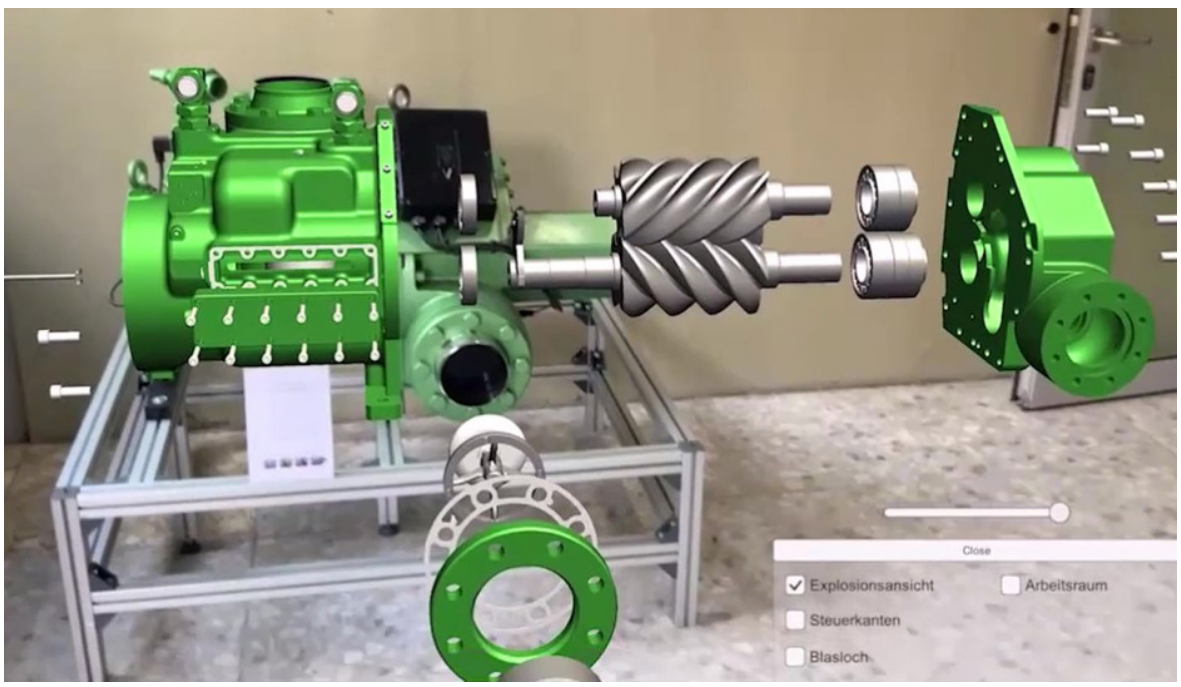

Abb. 11 AR-Funktion zur Explosionsdarstellung

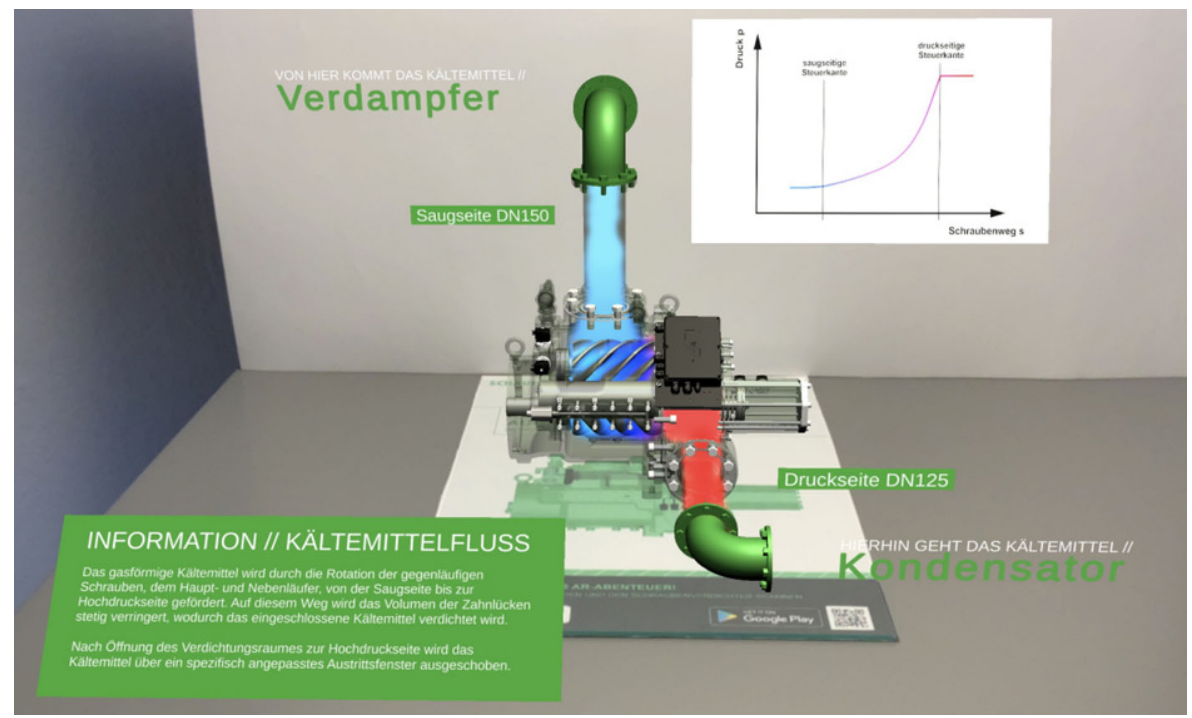

Abb. 12 AR-Funktion zur Animation des Kältemittelflusses und zur kontextabhängigen Informationsdarstellung

Mithilfe der Funktion Wissen wird der Lehrinhalt der VR-Anwendung in Form von Multiple-Choice abgefragt. Damit lässt sich das erlernte Wissen prüfen und ggf. bei Verständnisschwierigkeit der Inhalt so oft wie erforderlich wiederholen. 


\subsection{Evaluierung der AR-Anwendung zur Visualisierung des Aufbaus und der Funktion eines Schraubenverdichters im Schulungs- und Trainingsbereich durch BITZER}

Eine erste Projektarbeitsgruppe der Hochschule Karlsruhe erstellte aus den von BITZER bereitgestellten 3D-CAD-Daten erste Modelle. Die im Anschluss daran erfolgte Bachelorarbeit verfeinerte das Thema bis hin zur Fertigstellung einer App, die für die entsprechenden Plattformen vom Benutzer per QR-Code auf Flyern und Aufstellern heruntergeladen werden kann. Das ermöglicht, den Verdichter im eigenen Tempo kennenzulernen und seine Komponenten zu erkunden. Je nach Interesse und Vorkenntnis gibt es verschiedene Detaillierungsgrade, so dass für jeden Anwender etwas dabei ist. Auch eine kleine Wissensabfrage kann eingeblendet werden - ebenso eine Explosionszeichnung der Einzelkomponenten, der Fluid-(Kältemittel-)Fluss und maschinenbezogene Diagramme - wie das Einsatzgrenzendiagramm. Insgesamt bietet das digitale Schnittmodell viel mehr Informationen und Erläuterungen als reale Schnittmodelle abdecken können. Darüber hinaus steht das Modell quasi überall zur Verfügung und kann jederzeit in Onlineseminare, Präsentationen, Kunden- und Lieferantentermine integriert werden. Ein entscheidender Vorteil ergibt sich auch mit Blick auf die Masse von realen Modellen. Grundsätzlich funktioniert die AR-App im Targeting auch mit kleineren, deutlich handlicheren Kunststoffmodellen - zum Beispiel aus dem 3-D-Drucker. Somit kann gerade auch im Bereich des Marketings noch ein gewisser Anreiz geschaffen werden.

Die Modelle und Inhalte sind anpassbar - somit bietet die Plattform auch zukünftigen Entwicklungen Raum und Anpassungsmöglichkeiten. Sie ersetzen die heutige Bedienungsanleitung. Die Flexibilität und die Einsatzmöglichkeiten sind vielfältiger Art und bieten die Möglichkeit auch interessierten Schulen/Hochschulen und anderen Institutionen und Interessierten, Modelle ohne größeren Aufwand zukommen zu lassen. Wissensabfragen und Gamification sind genauso möglich, wie einfache Erklärungen. Insgesamt können AR-Schnittmodelle als Erfolg und würdiger Ersatz klassischer Schnittmodelle gesehen werden. Wie hier dargestellt, bieten sie einen großen Mehrwert. Sie geben Anreiz, sich für die Kälte- und Klimatechnik zu begeistern.

\section{Erfahrungsbericht}

\subsection{Erfahrungsbericht von Rosenbauer Karlsruhe}

Die Kooperationsprojekte mit der Hochschule Karlsruhe haben einen guten ersten Einblick in die Möglichkeiten und Chancen von AR gegeben. Basierend auf die umgesetzten AR-Projekte sind bereits weitere Ideen entwickelt worden, in denen AR eine Schlüsselrolle einnehmen wird. Die zunehmende Verbreitung von ARfähigen Smartphones wird hier ihr übriges tun, um diese Technologie einem zunehmenden Anwenderkreis zur Verfügung zu stellen. Genau dieser Umstand wird die Verbreitung von AR-Anwendungen beflügeln, mit der Konsequenz, dass sich ARAktivitäten im Bereich Marketing und Vertrieb lohnen werden. 


\subsection{Erfahrungsbericht von BITZER}

Die bis heute umgesetzten Projekte übertreffen hinsichtlich ihrer Ausführung und der Zeit, in der sie realisiert wurden, in positiver Weise jegliche Erwartungen und bieten in Zukunft sicherlich noch ungeahnte Potenziale - vor allem wenn die Prozesse weiter standardisiert und automatisiert werden. Laufende AR-Projekte finden durch die Zweisprachigkeit Anklang in BITZER Niederlassungen weltweit. Durch den Einsatz standardisierter Software die Inhalte jederzeit erweitert und optimiert werden. Fest steht, digitale Schnittmodelle können deutlich schneller und kostengünstiger erstellt werden. AR bietet enorme Potenziale technisch komplexe oder auch gegebenenfalls für den User gefährliche und nicht sichtbare Lehrinhalte auf spielerische, aber doch realitätsnahe Weise zu vermitteln. Simulation und AR bieten ähnlich der ergänzenden Ausbildung von Piloten in der Luftfahrt im technischen und industriellen Umfeld großartige Möglichkeiten. In der Zukunft wird diesen digitalen Anwendungen mit Sicherheit eine noch größere Bedeutung in der Ausbildung, Weiterbildung und auch in anderen Formen der Wissensvermittlung zukommen.

\subsection{Erfahrungsbericht im Rahmen der Lehre an der Hochschule Karlsruhe}

Die AR-App zur Visualisierung des Aufbaus und der Funktion eines Schraubenverdichters und daraus einfach zu extrahierende Videos werden bereits in den Vorlesungen zu den Grundlagen der Kältetechnik und der Verdichtertechnologie an der Hochschule Karlsruhe eingesetzt. Die Rückmeldung der Studierenden zu diesen neuen Formaten ist ausgesprochen positiv und untermauert den Ansatz einer modernen, digital unterstützten Lehre - auch unter den aktuellen Voraussetzungen der Corona-Pandemie. Als angenehmer Nebeneffekt wird das Image von in die Jahre gekommenen (leider häufig verstaubten) Schnittmodellen dramatisch aufgebessert und die Akzeptanz aus Sicht der Studierenden deutlich gesteigert. Anhand der Möglichkeiten der Darstellung über AR können den Studierenden auch schwierig verständliche Inhalte gut erklärt werden. Das Interesse sowie die Aufmerksamkeit lassen sich allein durch die Verwendung von digitalen Hilfsmitteln - zum Beispiel dem eigenen Mobiltelefon in der Lehrveranstaltung - deutlich steigern.

Funding Open Access funding enabled and organized by Projekt DEAL.

Open Access Dieser Artikel wird unter der Creative Commons Namensnennung 4.0 International Lizenz veröffentlicht, welche die Nutzung, Vervielfältigung, Bearbeitung, Verbreitung und Wiedergabe in jeglichem Medium und Format erlaubt, sofern Sie den/die ursprünglichen Autor(en) und die Quelle ordnungsgemäß nennen, einen Link zur Creative Commons Lizenz beifügen und angeben, ob Änderungen vorgenommen wurden.

Die in diesem Artikel enthaltenen Bilder und sonstiges Drittmaterial unterliegen ebenfalls der genannten Creative Commons Lizenz, sofern sich aus der Abbildungslegende nichts anderes ergibt. Sofern das betreffende Material nicht unter der genannten Creative Commons Lizenz steht und die betreffende Handlung nicht nach gesetzlichen Vorschriften erlaubt ist, ist für die oben aufgeführten Weiterverwendungen des Materials die Einwilligung des jeweiligen Rechteinhabers einzuholen.

Weitere Details zur Lizenz entnehmen Sie bitte der Lizenzinformation auf http://creativecommons.org/ licenses/by/4.0/deed.de. 


\section{Literatur}

(2021) Augmented Reality or Mixed Reality? https://www.augmented-minds.com/en/augmented-reality/ what-is-augmented-reality/. Zugegriffen: 26. Mai 2021

xr4all (2021) What is XR? https://xr4all.eu/xr/. Zugegriffen: 16. Mai 2021

Capgemini Augmented and virtual reality in operations: a guide for investment. https://www.capgemini. com/research-old/augmented-and-virtual-reality-in-operations/. Zugegriffen: 25. Mai 2021

DigiCapital (2019) For AR/VR 2.0 to live, AR/VR 1.0 must die. January 15, 2019. https://www.digicapital.com/news/2019/01/for-ar-vr-2-0-to-live-ar-vr-1-0-must-die/. Zugegriffen: 12. Juni 2020

Dörner R, Broll W, Grimm P, Jung B (2013) Virtual and Augmented Reality (VR/AR): Grundlagen und Methoden der Virtuellen und Augmentierten Realität. Springer Vieweg, Wiesbaden

Fitzgerald M, Kruschwitz M, Bonnet D, Welch M (2014) Embracing digital technology: a new strategic imperative. MIT Sloan Manage Rev 55(2). https://www.academia.edu/28433565/Embracing_Digital_ Technology_A_New_Strategic_Imperative. Zugegriffen: 01.12.2021

Horvat N, Škec S, Martinec T, Lukačević F, Perišić M (2019) Comparing virtual reality and desktop interface for reviewing 3D CAD models. International Conference on Engineering Design, Delft

Horvath I, Sudar A (2018) Factors contributing to the enhanced performance of the maxwhere $3 \mathrm{~d}$ vr platform in the distribution of digital information. Acta Polytech Hungarica 15(3):149-173

Liu Y, Lather J, Messner J (2014) Virtual reality to support the integrated design process: a retrofit case study. International Conference on Computing in Civil and Building Engineering, Orlando

Milgram P, Takemura H, Utsumi A, Kishino F (1994) Augmented reality: a class of displays on the realityvirtuality continuum. SPIE 2351: (Telemanipulator and Telepresence Technologies): 282-292

Nee AYC, Ong SK, Chryssolouris G, Mourtzis D (2012) Augmented reality applications in design and manufacturing. CIRP Annals 61(2):657-679

Schellinger J, Tokarski KO, Kissling-Näf I (2019) Digital Transformation und Unternehmensführung: Trend und Perspektiven für die Praxis. Springer Gabler, 